\title{
Low-intensity pulsed ultrasound promotes apoptosis and inhibits angiogenesis via p38 signaling-mediated endoplasmic reticulum stress in human endothelial cells
}

\author{
ZHONGPING SU ${ }^{1}$, TIANHUA XU ${ }^{1}$, YAQING WANG ${ }^{1}$, XIASHENG GUO $^{2}$, JUAN TU $^{2}$, \\ DONG ZHANG ${ }^{2}$, XIANGQING KONG ${ }^{1,3}$, YANHUI SHENG ${ }^{1}$ and WEI SUN ${ }^{1,3}$
}

\author{
${ }^{1}$ Department of Cardiology, The First Affiliated Hospital of Nanjing Medical University, Nanjing, Jiangsu 210029; \\ ${ }^{2}$ Key Laboratory of Modern Acoustics, Department of Physics, Collaborative Innovation Center of Advanced Microstructure, \\ Nanjing University, Nanjing, Jiangsu 210093; ${ }^{3}$ Department of Clinical Medicine, Collaborative Innovation Center for \\ Cardiovascular Disease Translational Medicine, Nanjing Medical University, Nanjing, Jiangsu 211166, P.R. China
}

Received July 29, 2018; Accepted March 26, 2019

DOI: $10.3892 / \mathrm{mmr} .2019 .10136$

\begin{abstract}
Aberrant increase in angiogenesis contributes to the progression of malignant solid tumors. An alternative anti-angiogenesis therapy is critical for cancer, since the current anti-angiogenesis drugs lack specificity for tumor cells. In the present study, the effects and mechanisms of low-intensity pulsed ultrasound (LIPUS) on human umbilical vein endothelial cells (HUVECs) and human microvascular endothelial cells (HMECs) were investigated, and the therapeutic potential of this technology was assessed. HUVECs and HMECs were treated with LIPUS $(0.5 \mathrm{MHz}$; $210 \mathrm{~mW} / \mathrm{cm}^{2}$ ) for $1 \mathrm{~min}$ and cultured for $24 \mathrm{~h}$. Flow cytometry and Cell Counting Kit- 8 assays demonstrated that LIPUS treatment at a dose of $210 \mathrm{~mW} / \mathrm{cm}^{2}$ promoted apoptosis and decreased the viability in HUVECs and HMECs. Real-time
\end{abstract}

Correspondence to: Dr Wei Sun or Professor Yanhui Sheng, Department of Cardiology, The First Affiliated Hospital of Nanjing Medical University, 300 Guangzhou Road, Nanjing, Jiangsu 210029, P.R. China

E-mail: weisun7919@njmu.edu.cn

E-mail: yhsheng@njmu.edu.cn

Abbreviations: LIPUS, low-intensity pulsed ultrasound; HUVECs, human umbilical vein endothelial cells; HMECs, human microvascular endothelial cells; HIFU, high-intensity focused ultrasound; MAPK, mitogen-activated protein kinase; ERK, extracellular signal-regulated kinase; JNK, c-Jun N-terminal kinase; ATF-4, activating transcription factor-4; eIF2 $\alpha$, eukaryotic initiation factor $2 \alpha$; p-, phosphorylated; ER, endoplasmic reticulum; CCK-8, Cell Counting Kit-8; RTCA, real-time cell analysis; CHOP, C/EBP homologous protein

Key words: low-intensity pulsed ultrasound, apoptosis, p38 mitogen-activated protein kinase, eIF $2 \alpha$, eukaryotic initiation factor $2 \alpha$ /activating transcription factor-4, human umbilical vein endothelial cells, human microvascular endothelial cells cell analysis also revealed that LIPUS did not affect the proliferation or migration of HUVECs. An endothelial cell tube formation assay indicated that LIPUS treatment inhibited the angiogenic ability of HUVECs and HMECs. Furthermore, LIPUS increased the protein levels of the apoptosis-associated cleaved Caspase- 3 and decreased the B-cell lymphoma-2 levels. LIPUS increased the phosphorylation of p38 mitogen-activated protein kinase (MAPK), and the levels of endoplasmic reticulum (ER) stress-associated markers, including activating transcription factor-4 (ATF-4) and phosphorylated eukaryotic initiation factor $2 \alpha$ (eIF2 $\alpha$ ). The p38 inhibitor SB203580 reversed the pro-apoptotic and anti-angiogenic effects of LIPUS in cells. Finally, inhibition of p38 decreased the LIPUS-induced elevation of p-eIF2 $\alpha$ and ATF-4 levels. Taken together, these results suggested that LIPUS promoted apoptosis and inhibited angiogenesis in human endothelial cells via the activation of p38 MAPK-mediated ER stress signaling.

\section{Introduction}

Malignant cancer poses a major threat to human life and health. Current treatment methods, including surgery, chemotherapy and radiotherapy, aim to directly kill tumor cells or induce cell s apoptosis (1). However, chemotherapy and radiotherapy have toxic side effects, since they induce the death of a large number of bone marrow cells and other normal dividing cells, in addition to tumor cells (2). Therefore, these methods have numerous limitations in clinical applications.

Angiogenesis is the process of new blood vessel formation. Tumor growth depends on continuous and extensive angiogenesis, which provides nutrients and oxygen to the tumor tissues. Distant metastasis of tumor cells occurs via blood vessels. Therefore, targeting tumor angiogenesis has become an important alternative therapy for the control of tumor growth and metastasis $(3,4)$. Anti-angiogenesis therapies that target the vascular endothelial system are widely used for cancer treatment (5-7). However, existing anti-angiogenic agents lack specificity, and thus impair angiogenesis in tumor and 
normal tissues $(8,9)$. Certain tumors have even been reported to develop resistance to anti-angiogenesis drugs (8). An alternative non-drug therapy that overcomes these limitations is necessary for anti-angiogenesis therapy.

Recent advances in ultrasound technology have led to a better understanding of the biological effects of ultrasound. The high-intensity focused ultrasound (HIFU) technology has presented promising results in the treatment of various cancer types, such as pancreatic cancer (10). HIFU is a non-invasive technique that delivers focused, high-intensity ultrasound energy $\left(\geq 3 \mathrm{~W} / \mathrm{cm}^{2}\right)$ to a specific area of the body to instantly raise its temperature to approximately $65-70^{\circ} \mathrm{C}$, which results in irreversible cell death through coagulation necrosis (11). These thermal effects are considered to be the primary mechanism by which HIFU ablates tumor cells $(12,13)$. However, specific ablation of tumor cells remains a challenge, given that repeated HIFU treatments have been reported to cause damage to healthy tissues that are in the path of the acoustic beam $(14,15)$.

Low-intensity pulsed ultrasound (LIPUS) delivers ultrasound waves at a much lower intensity (usually $<300 \mathrm{~mW} / \mathrm{cm}^{2}$ ) and at a lower frequency range of $20 \mathrm{kHz}$ to $1 \mathrm{MHz}$ compared with HIFU; therefore, it does not exhibit hyperthermal effects. LIPUS has been widely used for fracture healing, wound healing in different tissue types, inhibition of bacterial growth, enhanced drug delivery to the brain and in vitro thrombolysis (16-18). LIPUS induced selective apoptosis of brain tumor cells in rat brain glioma (19), without damage to the surrounding normal tissues, or any of the side effects that occur with radiation therapy and chemotherapy (20). However, the therapeutic potential of LIPUS for anti-angiogenesis therapy remains largely unknown.

In the present study, the effects of different doses of LIPUS on the proliferation, migration, apoptosis and angiogenesis of human endothelial cells, including human umbilical vein endothelial cells (HUVECs) and human microvascular endothelial cells (HMECs), were investigated. It was observed that a mean intensity of $210 \mathrm{~mW} / \mathrm{cm}^{2}$ significantly promoted apoptosis and inhibited angiogenesis via increased phosphorylation of p38 mitogen-activated protein kinase (MAPK) and endoplasmic reticulum (ER) stress signals. The study results suggested a potential role of LIPUS in anti-angiogenesis therapy.

\section{Materials and methods}

Culture of HUVECs and HMECs. HUVECs were purchased from the American Type Culture Collection (ATCC; cat. no. PCS-100-010; Manassas, VA, USA) and were incubated in Dulbecco's modified Eagle's medium (Gibco; Thermo Fisher Scientific, Inc., Waltham, MA, USA) containing $10 \%$ fetal bovine serum (Gibco; Thermo Fisher Scientific, Inc.), and $1 \%$ penicillin $(100 \mathrm{U} / \mathrm{ml})$ and streptomycin $(100 \mu \mathrm{g} / \mathrm{ml})$. HMECs were purchased from the ATCC (cat. no. CRL-3243) and cultured in endothelial cell medium (cat. no. 1001; ScienCell Research Laboratories, Inc., San Diego, CA, USA) containing 5\% (cat. no. 0025; ScienCell Research Laboratories, Inc.), $1 \%$ endothelial cell growth supplement (cat. no. 1052; ScienCell Research Laboratories, Inc) and $1 \%$ penicillin/streptomycin solution. The cells were maintained at $37^{\circ} \mathrm{C}$ in $5 \% \mathrm{CO}_{2}$. At $80-90 \%$ confluence, the cells were suspended at a concentration of $1 \times 10^{6}$ cells $/ \mathrm{ml}$ and plated on $6-\mathrm{cm}$ culture dishes. The cell suspension was exposed to LIPUS for $1 \mathrm{~min}$ and then subjected to various analyses. In addition, the effect of culture for $24 \mathrm{~h}$ with a specific inhibitor of p38 MAPK, SB203580 (4 $\mu \mathrm{M}$; Selleck Chemicals, Houston, TX, USA) subsequent to LIPUS exposure for $1 \mathrm{~min}$ was also examined.

LIPUS stimulation. LIPUS irradiation was performed using a set of ultrasound devices that included a signal generator (Agilent Technologies, Inc., Santa Clara, CA, USA), a wide-band power amplifier (Electronics and Innovation Ltd, Rochester, NY, USA) and a planar transducer (Chongqing Haifu Medical Technology Co., Ltd., Chongqing, China). The planar transducer was set at a frequency of $0.5 \mathrm{MHz}$, the voltage applied to the transducer was $44 \mathrm{~V}$, the ultrasonic intensity was $70-280 \mathrm{~mW} / \mathrm{cm}^{2}$, the number of cycles was 1,000-4,000 and the mean acoustic pressure was $0.5 \mathrm{MPa}$. Briefly, a 6-cm dish seeded with $1 \times 10^{6}$ cells was placed on top of the transducer (diameter size, $6 \mathrm{~cm}$ ), with degassed water between the dish and transducer. Subsequently, the cell suspension was exposed to LIPUS for $1 \mathrm{~min}$ and cultured for $24 \mathrm{~h}$. The control cell suspension was treated identically to the experimental group, with the exception of the LIPUS treatment. A temperature test paper (TMCHallcrest, Glenview, IL, USA) was adhered to the inner surface of the $6-\mathrm{cm}$ dish to measure the temperature. Details of the different doses of LIPUS used in the experiment are listed in Table I.

Flow cytometry analysis of cell apoptosis. The apoptosis rates of cells were analyzed by flow cytometry using an Annexin V-Alexa Fluor ${ }^{\circledR} 647$ detection kit (cat. no. FMSAV647-100, FCMACS Biotech Co. Ltd., Nanjing, China) according to the manufacturer's protocol. LIPUS-treated cells and control cells were seeded in 12-well plates at a density of $5 \times 10^{5}$ cells/well and harvested $24 \mathrm{~h}$ later. The cells were then centrifuged at $300 \mathrm{x} \mathrm{g}$ for $5 \mathrm{~min}$ at $4^{\circ} \mathrm{C}$. Following two washes with PBS, the cells were resuspended in $100 \mu \mathrm{l}$ of binding buffer. Subsequently, cells were stained with $5 \mu$ l Annexin V-Alexa Fluor ${ }^{\circledR} 647$ and $10 \mu$ l propidium iodide (PI) for $15 \mathrm{~min}$ in the dark prior to conducting the flow cytometry analysis (BD Biosciences, San Jose, USA). Early apoptotic cells (Annexin V-positive/PI-negative) were located in the lower right quadrant. Late apoptotic or necrotic cells (positive for Annexin V and PI) were located in the upper right quadrant. Live cells (negative for Annexin V and PI) were located in the lower left quadrant. Dead cells (Annexin V-negative/PI-positive) were located in the upper left quadrant (Fig. 1A and B). The early apoptosis rate was calculated based on the number of cells in the lower right quadrant following the manufacturer's protocol.

Cell Counting Kit-8(CCK-8) assay. The viability of HUVECs and HMECs was assessed using a CCK-8 assay (Dojindo Molecular Technologies, Inc., Kumamoto, Japan). Briefly, cells were seeded at a concentration of $8 \times 10^{3}$ cells/well in 96-well plates for $24 \mathrm{~h}$. Next, $10 \mu \mathrm{l} \mathrm{CCK-8} \mathrm{reagent} \mathrm{was} \mathrm{added}$ to each well, and the cells were incubated for an additional 2-4 h. The absorbance at $450 \mathrm{~nm}$ was then measured using a microplate reader. 
Table I. List of acoustic parameters used in the present study, all used at a frequency of $0.5 \mathrm{MHz}$.

\begin{tabular}{lccc}
\hline $\begin{array}{l}\text { No. } \\
\text { of cycles }\end{array}$ & $\begin{array}{c}\text { Ultrasonic } \\
\text { power }(\mathrm{W})\end{array}$ & $\begin{array}{c}\text { Ultrasonic intensity } \\
\left(\mathrm{mW} / \mathrm{cm}^{2}\right)\end{array}$ & $\begin{array}{c}\text { Temperature } \\
\left({ }^{\circ} \mathrm{C}\right)\end{array}$ \\
\hline 1,000 & 1.42 & 70 & 30 \\
2,000 & 2.80 & 140 & 35 \\
3,000 & 4.16 & 210 & 35 \\
4,000 & 5.54 & 280 & 38 \\
\hline
\end{tabular}

Real-time cell analysis (RTCA). RTCA, a label-free dynamic technology, was used in the current study to monitor the proliferation and migration of HUVECs in real time. To evaluate cell proliferation, cell suspensions (100 $\mu \mathrm{l} ; 2,000-3,000$ cells/well) were seeded in an E-Plate assay plate (ACEA Biosciences, Inc.; Agilent Technologies, Inc., Santa Clara, CA, USA), placed at room temperature on an ultra-clean bench for $30 \mathrm{~min}$ and were monitored continuously for $48 \mathrm{~h}$ using the RTCA TP System (ACEA Biosciences, Inc.; Agilent Technologies, Inc.).

In order to monitor cell migration, $100 \mu 1$ serum-free cell suspension (3,000 cells/well) was added to the upper chamber of a CIM-Plate test plate (ACEA Biosciences, Inc.; Agilent Technologies, Inc.), while serum-containing medium was added to the lower chamber. The set-up was placed at room temperature on an ultra-clean bench for $30 \mathrm{~min}$ and then placed on a test bench to monitor cell migration for $24 \mathrm{~h}$ using the RTCA TP System (ACEA Biosciences, Inc.; Agilent Technologies, Inc.).

Endothelial cell tube formation assay. To examine the effect of LIPUS on angiogenesis in vitro, a capillary-like tube formation assay was performed. Matrigel matrix (cat. no. 354234; Corning Incorporated, Corning, NY, USA) was pipetted into pre-chilled 96 -well plates (50 $\mu \mathrm{l}$ Matrigel/well) and polymerized at $37^{\circ} \mathrm{C}$ for $30-60 \mathrm{~min}$. Next, HUVECs $\left(2 \times 10^{4}\right.$ cells/well) or HMECs $\left(2.5 \times 10^{4}\right.$ cells/well $)$ in complete media were seeded in Matrigel-coated plates. After $8 \mathrm{~h}$ of incubation, images of the tubular structures were captured.

Western blotting. Following treatment, HUVECs cells were lysed using whole cell lysis buffer (cat. no. KGP250/KGP2100; Nanjing Keygen Biotech Co., Ltd., Nanjing, China) containing $1 \%$ PMSF on ice for $30 \mathrm{~min}$ to extract total protein. The protein concentration was determined using a Bicinchoninic Acid Protein Quantification kit (cat. no. P0009; Beyotime Institute of Biotechnology, Beijing, China). Equal amounts of protein (30 $\mu \mathrm{g} /$ lane) were separated via 8-15\% SDS-PAGE. Proteins were then transferred onto polyvinylidene fluoride membranes (EMD Millipore, Billerica, MA, USA) and blocked with $5 \%$ bovine serum albumin (MP Biomedicals, LLC, Santa Ana, CA, USA) for $2 \mathrm{~h}$ at room temperature. Subsequently, the membranes were probed overnight at $4^{\circ} \mathrm{C}$ with primary antibodies recognizing the following antigens: $\beta$-tubulin (cat. no. 2128), p38 (cat. no. 8690), phosphorylated (p)-p38 (cat. no. 4511), extracellular signal-regulated kinase (ERK; cat. no. 4695), p-ERK (cat. no. 4370), c-Jun N-terminal kinase (JNK; cat. no. 9252), p-JNK (cat. no. 4668), B-cell lymphoma-2
(Bcl-2; cat. no. 2876), activating transcription factor-4 (ATF-4; cat. no. 11815), phosphorylated eukaryotic initiation factor $2 \alpha$ (p-eIF2 $\alpha$; cat. no. 3597), Bcl-2-associated X protein (Bax; cat. no. 2772), cleaved Caspase-3 (cat. no. 9661) and light chain 3B (LC3B; cat. no. 2775). All primary antibodies were purchased from Cell Signaling Technology, Inc., (Danvers, MA, USA) and used at a dilution of 1:1,000. Membranes were then washed and incubated with corresponding horseradish peroxidase-labeled goat anti-rabbit (1:5,000, PV-9003; ZSGB-Bio, China) or goat anti-mouse secondary antibodies (1:5,000, ZB-2305; ZSGB-Bio, China) for $2 \mathrm{~h}$ at room temperature. Finally, the target proteins were detected using an enhanced chemiluminescence kit (cat. 34096; Thermo Fisher Scientific, Inc.) and exposed on a ChemiDoc MP imager (Bio-Rad, California, USA). Bands were normalized to $\beta$-tubulin, and protein levels were quantified by ImageJ software (v.1.8.0; National Institutes of Health, Bethesda, MD, USA).

Statistical analysis. Data are expressed as the mean \pm standard error of the mean from three independent experiments. Treatment group values were compared with control values using GraphPad Prism software (v.6.0; GraphPad Software, Inc., La Jolla, CA, USA). Comparisons between two observations were assessed by unpaired Student's t-test. One-way analysis of variance was used, followed by the Bonferroni post-hoc test for multiple comparisons. $\mathrm{P}<0.05$ was considered to indicate a statistically significant difference.

\section{Results}

LIPUS promotes the apoptosis and inhibits the viability of HUVECs and HMECs. To study the effects of LIPUS on the apoptosis of HUVECs and HMECs, the cells were treated with different doses of ultrasonic intensities (Table I). Flow cytometry analysis demonstrated that a single treatment of LIPUS for a duration of $1 \mathrm{~min}$ promoted early apoptosis in HUVECs and HMECs in a dose-dependent manner (Fig. 1A-D). Ultrasound intensities as low as $140 \mathrm{~mW} / \mathrm{cm}^{2}$ promoted early apoptosis compared with the control group. The early apoptosis rate was significantly higher at the dose of 140,210 and $280 \mathrm{~mW} / \mathrm{cm}^{2}$ in LIPUS-treated cells compared with that observed in control cells (Fig. 1C and D). Additionally, the rates of late apoptosis and cell death were significantly increased when the dose was increased to $280 \mathrm{~mW} / \mathrm{cm}^{2}$ (data not shown).

To analyze the thermal effects of LIPUS, the present study also measured the temperature of the cell suspension treated with LIPUS at doses ranging between 70 and $280 \mathrm{~mW} / \mathrm{cm}^{2}$. The results revealed that the temperature increased from $30^{\circ} \mathrm{C}$ to $35^{\circ} \mathrm{C}$ at a LIPUS dose range of $70-210 \mathrm{~mW} / \mathrm{cm}^{2}$, whereas the temperature reached $38^{\circ} \mathrm{C}$ at a dosage intensity of $280 \mathrm{~mW} / \mathrm{cm}^{2}$ (Table I). To investigate the effects of LIPUS under normal physiological temperature conditions $\left(<37.5^{\circ} \mathrm{C}\right)$, a mean dose intensity of $210 \mathrm{~mW} / \mathrm{cm}^{2}$ was selected for use in further experiments.

Next, the effects of LIPUS on HUVEC and HMEC viability were assessed using a CCK-8 assay. It was demonstrated that LIPUS treatment reduced the viability of these cells in a dose-dependent manner, with the lowest viability observed at a dosage intensity of $280 \mathrm{~mW} / \mathrm{cm}^{2}$ (Fig. 1E and F). Furthermore, the effects of LIPUS on the proliferation and migration of 

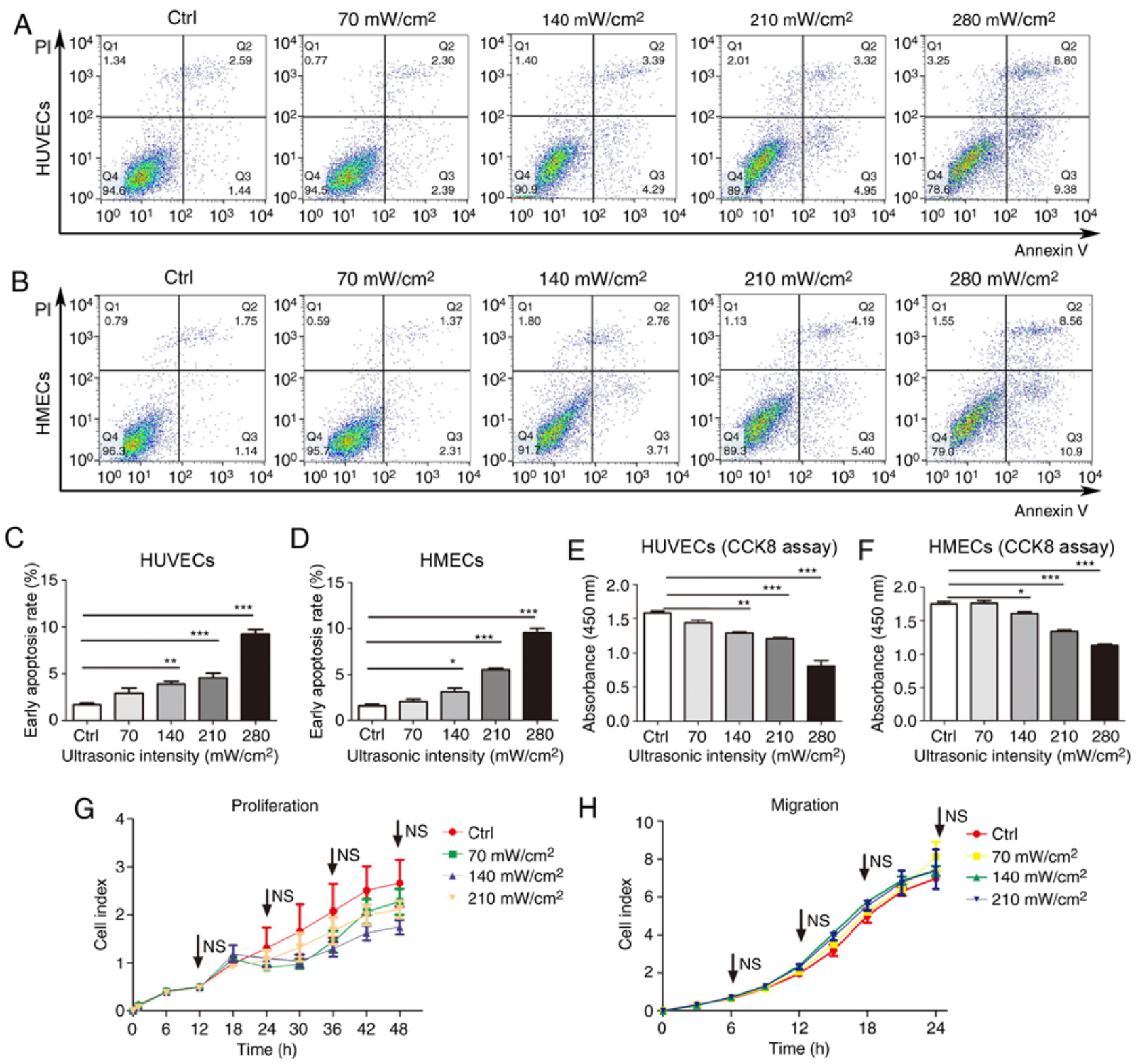

Figure 1. LIPUS promoted apoptosis and inhibited cell viability in HUVECs and HMECs. Flow cytometry analysis of apoptosis rate in (A) HUVECs and (B) HMECs treated with different dose of LIPUS (70-280 $\mathrm{mW} / \mathrm{cm}^{2}$ ). Quantification of early apoptosis in (C) HUVECs and (D) HMECs. Viability of (E) HUVECs and (F) HMECs treated with different doses of LIPUS $\left(70-280 \mathrm{~mW} / \mathrm{cm}^{2}\right)$ was assessed by CCK-8 assay. Real-time monitoring of the (G) proliferation and $(\mathrm{H})$ migration of HUVECs by real-time cell analysis. All values are expressed as the mean \pm standard error of three independent experiments. ${ }^{*} \mathrm{P}<0.05,{ }^{* *} \mathrm{P}<0.01$ and ${ }^{* * *} \mathrm{P}<0.001$. LIPUS, low-intensity pulsed ultrasound; HUVECs, human umbilical vein endothelial cells; HMECs, human microvascular endothelial cells; CCK-8, Cell Counting Kit-8; Ctrl, control; NS, not significant.

HUVECs were analyzed. The cells were treated with different doses of LIPUS, and then cell proliferation and migration were measured at 48 and $24 \mathrm{~h}$, respectively. The results revealed that dose intensities of $70-210 \mathrm{~mW} / \mathrm{cm}^{2}$ did not markedly affect the proliferation and migration of LIPUS-treated HUVECs as compared with untreated cells (Fig. $1 \mathrm{G}$ and $\mathrm{H}$ ).

LIPUS inhibits angiogenesis in HUVECs and HMECs. To determine the potential anti-angiogenic effects of LIPUS in HUVECs and HMECs, an in vitro angiogenesis assay was performed. After $8 \mathrm{~h}$ of incubation, LIPUS treatment was observed to significantly inhibit tube formation in treated cells as compared with that observed in untreated cells (Fig. 2A-D).
LIPUS regulates the expression of apoptosis-associated proteins. To examine the mechanism underlying the LIPUS-induced apoptosis, the levels of apoptosis marker proteins were measured in LIPUS-treated HUVECs. The protein level of cleaved Caspase-3 was significantly increased in cells treated with LIPUS at a dose of $210 \mathrm{~mW} / \mathrm{cm}^{2}$ as compared with that in control cells. LIPUS-treated cells also exhibited a marked reduction in the levels of the apoptosis inhibitor, $\mathrm{Bcl}-2$, and a higher $\mathrm{Bax} / \mathrm{Bcl}-2$ ratio, which is usually used for the measurement of the apoptotic potential of cells (21). In addition, the protein levels of the two forms of the autophagy-marker protein LC3B (namely LC3-I and-II) were also measured, and it was observed that these proteins were not markedly affected by LIPUS treatment (Fig. 3A and B). 

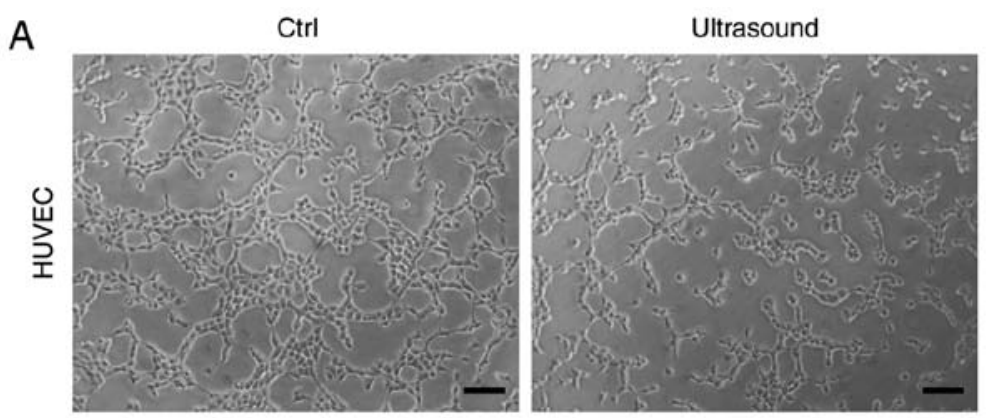

B
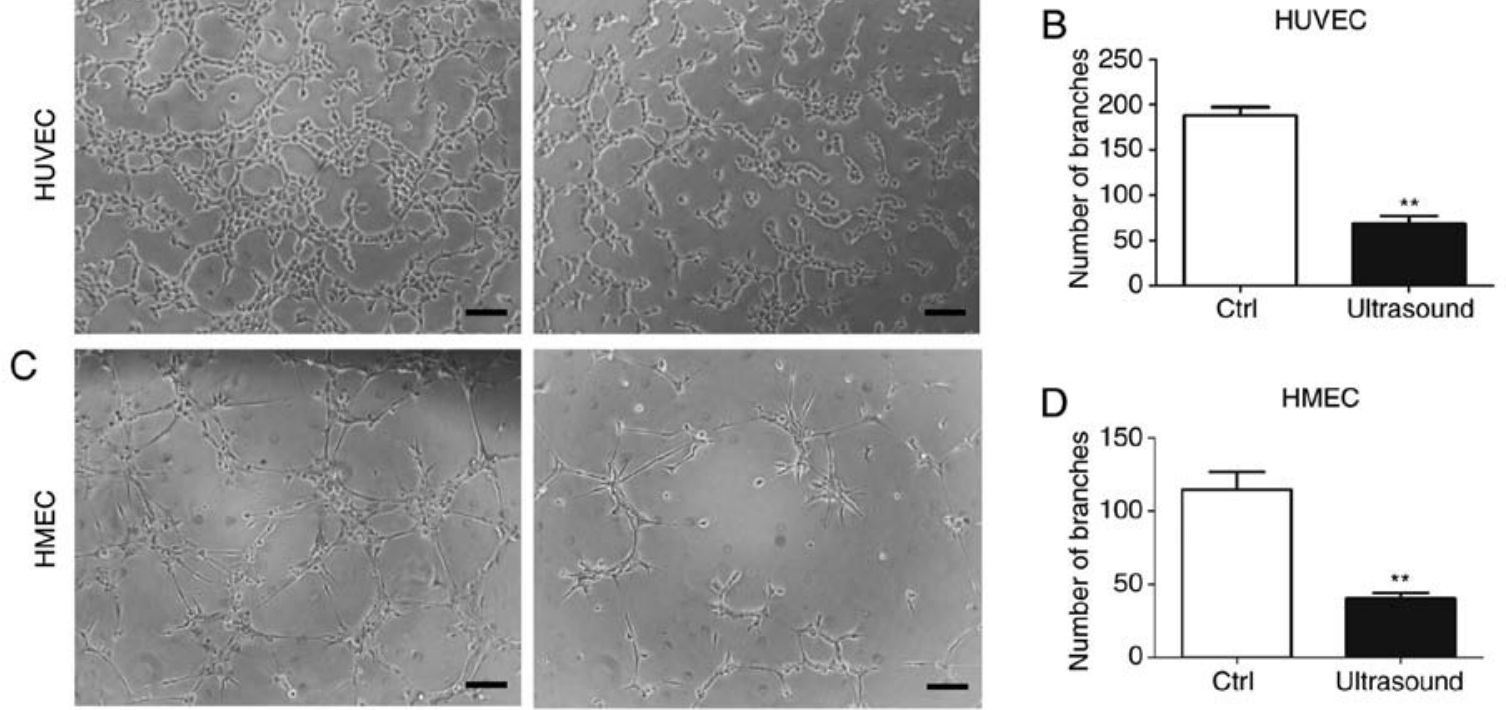

Figure 2. Effects of LIPUS on HUVEC and HMEC tube formation. Following incubation for $8 \mathrm{~h}$, images (magnification, 10x) of tube-like structures in the Ctrl and LIPUS-treated cells were obtained using a microscope camera, and quantification of branches was performed with ImageJ software. (A) Images of HUVEC tube formation and (B) quantified results. (C) Images of HMEC tube formation and (D) quantified results. All values are expressed as the mean \pm standard error of three independent experiments (scale bars $=100 \mu \mathrm{m}$ ). ${ }^{* *} \mathrm{P}<0.01 \mathrm{vs}$. Ctrl group. LIPUS, low-intensity pulsed ultrasound; HUVECs, human umbilical vein endothelial cells; HMECs, human microvascular endothelial cells; Ctrl, control.

LIPUS increases $p 38$ MAPK phosphorylation and activates ER stress signaling. To investigate the molecular mechanisms of LIPUS-induced apoptosis in HUVECs, the activated MAPK members were analyzed using western blotting. It was observed that LIPUS-treated cells exhibited significantly higher levels of p-p38 compared with the control cells. By contrast, LIPUS treatment resulted in decreased phosphorylation of ERK in these cells, while no significant difference was observed in the phosphorylation levels of JNK between treated and untreated cells (Fig. 3C and D).

ER stress signals are known to serve a major role in cell apoptosis (22). To investigate whether LIPUS-induced apoptosis in HUVECs was mediated by the ER stress signaling pathway, the levels of key ER stress proteins, including ATF-4 and p-eIF $2 \alpha$, were also measured. The data indicated that the levels of ATF-4 and p-eIF2 $\alpha$ were markedly increased in LIPUS-treated cells when compared with those in control cells (Fig. 3E and F).

Inhibition of $p 38$ phosphorylation rescues the pro-apoptotic and anti-angiogenic effects of LIPUS. To determine whether the pro-apoptotic effect of LIPUS on HUVECs was dependent on p38 phosphorylation, the specific p38 MAPK inhibitor SB203580 was used to decrease p-p38 levels in LIPUS-treated HUVECs. Flow cytometry analysis revealed that $\mathrm{p} 38$ inhibition in LIPUS-treated HUVECs resulted in reduced levels of apoptosis compared with those in inhibitor-free,LIPUS-treated HUVECs (Fig. 4A and B). Western blotting also revealed that p38 inhibition reversed the LIPUS-induced changes in the expression levels of Bcl-2 and cleaved Caspase-3 in HUVECs (Fig. 4C and D). The effect of p38 inhibition on angiogenesis subsequent to LIPUS treatment was examined. HUVECs and HMECs treated with both LIPUS and SB203580 exhibited recovered tube formation compared with the LIPUS-treated cells alone (Fig. 4E-H). These findings suggested that $\mathrm{p} 38$ phosphorylation served a key role in mediating the anti-angiogenic effect of LIPUS.

The effect of p38 inhibition on ERK and ER stress proteins was also examined in LIPUS-treated HUVECs. Western blotting revealed that p38 inhibition reversed the LIPUS-induced effects, leading to a significant increase of p-ERK levels (Fig. 5A and B), and marked reduction of ATF-4 and p-eIF2 $\alpha$ levels (Fig. 5C and D) as compared with the LIPUS-treated cells alone.

\section{Discussion}

LIPUS is increasingly used for various therapeutic purposes, including tumor ablation, bone repair, targeted drug delivery and chemotherapy (23). The mechanical signal of LIPUS is translated into a number of cellular effects, including anti-angiogenesis, anti-inflammatory responses and cytotoxicity (23-25). Therefore, LIPUS is an attractive, non-invasive and non-toxic option for standard oncology treatments, such as surgery, radiotherapy, gene therapy and chemotherapy (10). HUVECs are derived from endothelial vein tissues of the umbilical cord, while HMECs are derived from dermal endothelial tissues. These two cell lines are useful in vitro models for the study of endothelial functions, such as angiogenesis. Studies have reported that low-intensity ultrasound with microbubbles induces the evident microvascular damage in tumors, mainly due to inertial cavitation, which can cause cell necrosis or apoptosis $(26,27)$. Acoustic cavitation can lead to mechanical damage of small blood vessels due to the expansion and collapse caused by oscillation of the microbubbles (28). These previous studies have tested different LIPUS parameters and radiation times. In the present study, the effect of LIPUS treatment on HUVECs and HMECs was examined 

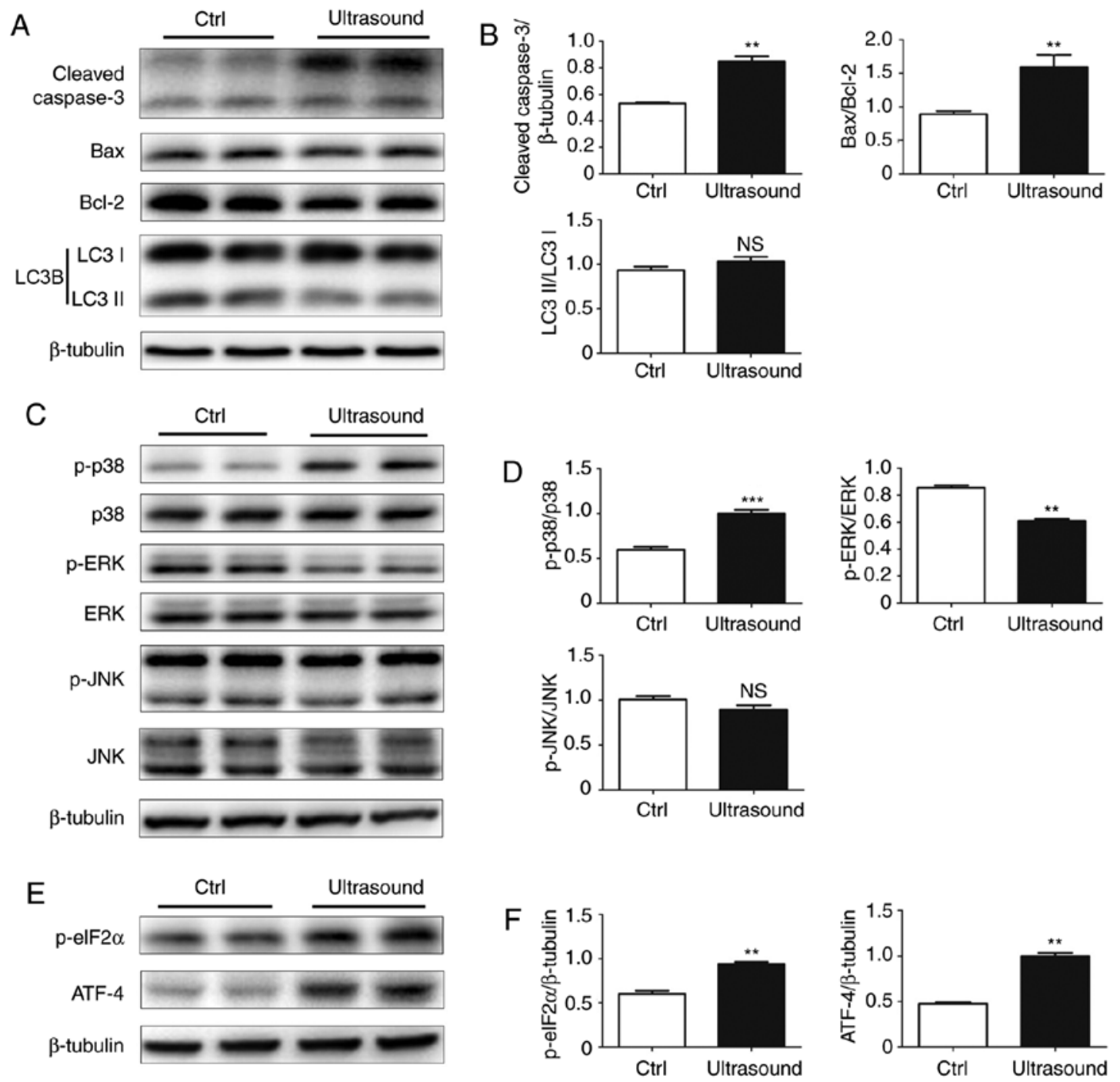

Figure 3. Low-intensity pulsed ultrasound regulated the expression of apoptosis-associated proteins. (A) Cleaved Caspase-3, Bcl-2, Bax and LC3B levels, measured by western blotting. (B) Quantification of the relative levels of cleaved Caspase-3, Bax and LC3-II, which were respectively normalized to $\beta$-tubulin, Bcl-2 and LC3-I levels. (C) Phosphorylation and total protein levels of p38, ERK and JNK were assessed by western blotting. (D) Quantification of p-p38, p-ERK and p-JNK protein levels, normalized to their total protein levels. (E) Protein levels of ATF-4 and p-eIF2 $\alpha$ were assessed by western blotting. (F) Quantification of ATF-4 and p-eIF2 $\alpha$ protein levels, normalized to $\beta$-tubulin. All values are expressed as the mean \pm standard error of three independent experiments. ${ }^{* *} \mathrm{P}<0.01$ and ${ }^{* * *} \mathrm{P}<0.001$, vs. Ctrl group. HUVECs, human umbilical vein endothelial cells; HMECs, human microvascular endothelial cells; Bcl-2, B-cell lymphoma-2; Bax, Bcl-2-associated X protein; LC3, light chain 3; ERK, extracellular signal-regulated kinase; JNK, c-Jun N-terminal kinase; ATF-4, activating transcription factor-4; eIF $2 \alpha$, eukaryotic initiation factor $2 \alpha$; Ctrl, control; p-, phosphorylated.

at ultrasound dose intensities of $70-280 \mathrm{~mW} / \mathrm{cm}^{2}$ for the same radiation time period, as previously reported (26-28). The results revealed that a larger dose of LIPUS directly inhibited cell viability and tube formation, and promoted apoptosis. The pro-apoptotic effect of LIPUS observed in the current study may be due to mechanical stimulation rather than inertial cavitation. As the temperature of cells treated with LIPUS at a dosage intensity of $210 \mathrm{~mW} / \mathrm{cm}^{2}$ was $35^{\circ} \mathrm{C}$ (close to the normal physiological temperature), possible thermal effects of LIPUS can be ruled out. The present in vitro study has highlighted the therapeutic potential of LIPUS in inhibiting angiogenesis and tumor growth via vascular endothelial cell apoptosis. Further in vivo studies are necessary to fully understand the effect of LIPUS on tumor angiogenesis.

The two main signaling pathways that regulate cell apoptosis are the extrinsic pathway, which is mediated by the activation of cell-surface death receptors by external signals, and the intrinsic pathway, which is activated by intracellular signals that cause mitochondrial damage $(29,30)$. Numerous factors, such as death receptor-mediated signaling molecules, anticancer drugs and growth factor inhibitors, can damage mitochondrial function and induce apoptosis (21). Bcl-2 is part of the Bcl-2 family of proteins that are well-known for their role in apoptosis regulation; this protein is localized in the mitochondrial outer membrane and serves a key role in cell survival by inhibiting pro-apoptotic molecules (31). Bax is a pro-apoptotic protein that is also a member of the Bcl-2 family and induces apoptosis via cytochrome $\mathrm{C}$-mediated cleavage of Caspase-3 (32). In the current study, the effect of LIPUS treatment on the expression levels of Bcl-2, Bax and cleaved Caspase-3 was assessed. Decreased expression of Bcl-2 and increased expression of cleaved Caspase-3 were observed in HUVECs in response to LIPUS treatment. Although no significant differences were observed in Bax levels between 
A

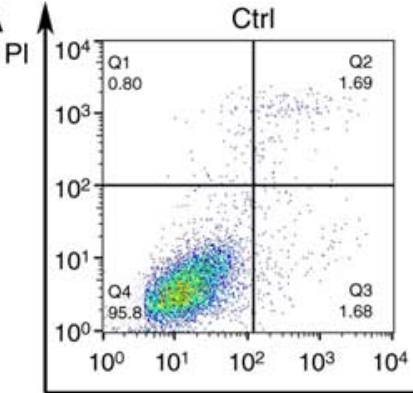

Ultrasound

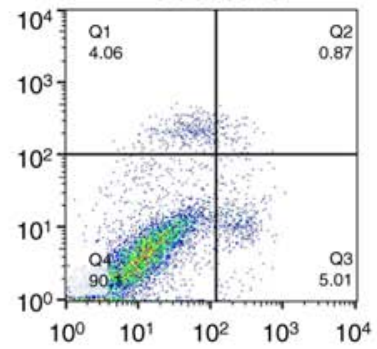

Ultrasound+SB203580

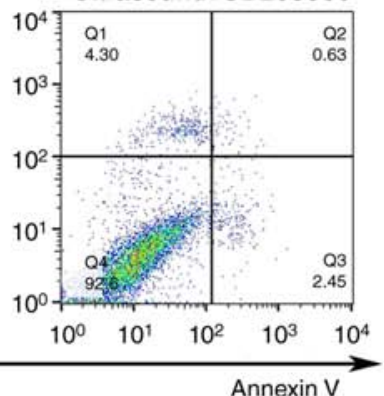

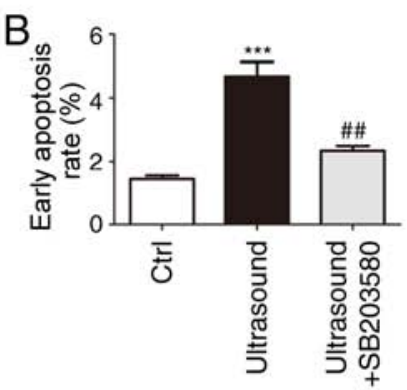
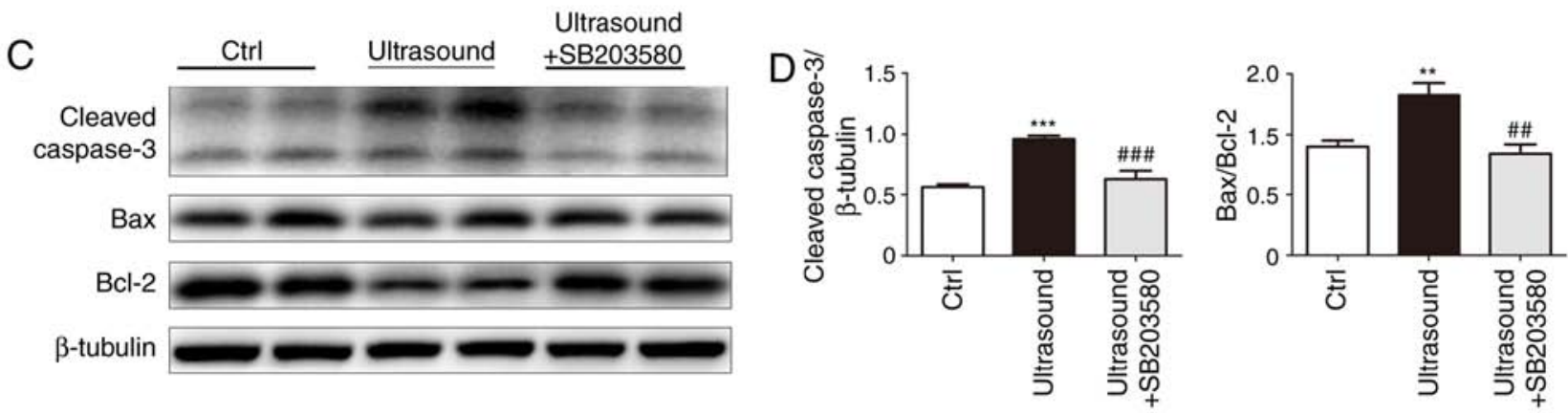

$\mathrm{F}$

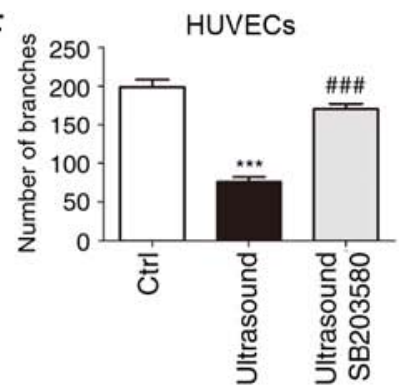

E

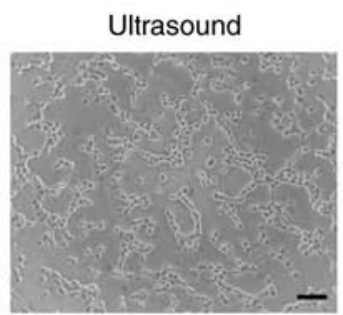

Ultrasound + SB203580
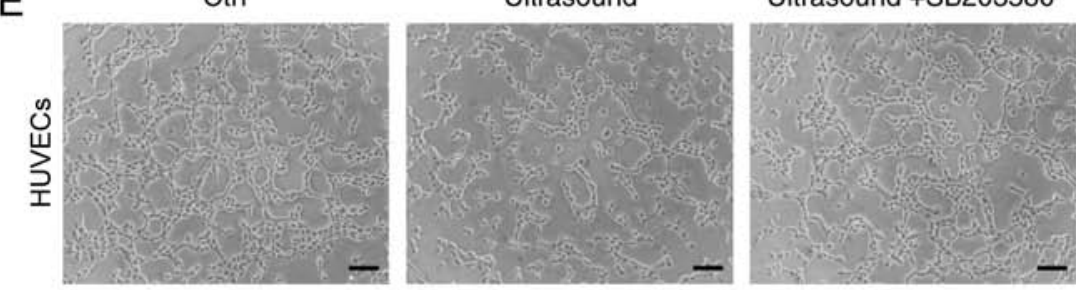

G
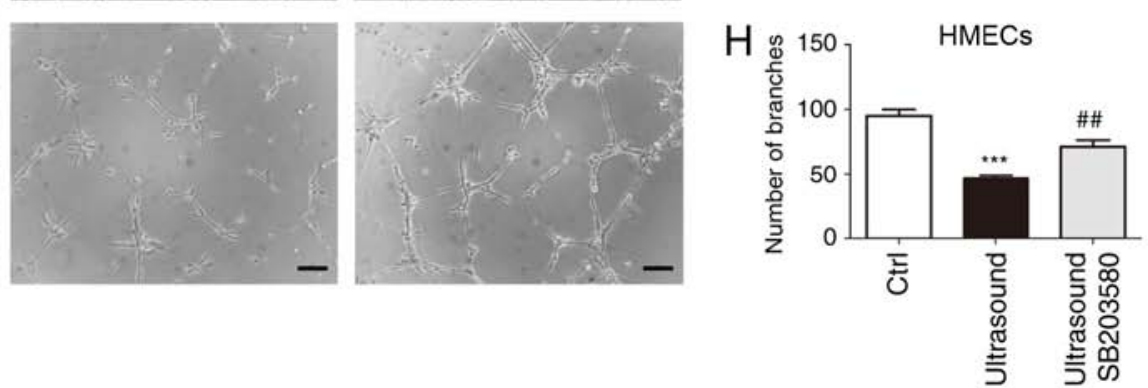

Figure 4. Inhibition of $\mathrm{p} 38$ phosphorylation rescued the pro-apoptotic and anti-angiogenic effects of low-intensity pulsed ultrasound. (A) Flow cytometry analysis of apoptosis rates in HUVECs treated with the p38 inhibitor SB203580. (B) Quantification of the percentage of early apoptotic HUVECs. (C) Western blots and (D) quantified protein levels of cleaved Caspase-3, Bcl-2 and Bax in HUVECs treated with S203580. (E) Images (magnification, 10x) of tube-like structures and (F) quantified results in HUVECs. (G) Images of tube-like structures and (H) quantified results in HMECs. Quantification of branches was conducted with ImageJ software. All values are expressed as the mean \pm standard error of three independent experiments (scale bars $=100 \mu \mathrm{m}) .{ }^{* * *} \mathrm{P}<0.01$ and ${ }^{* * * *} \mathrm{P}<0.001$, vs. Ctrl group; ${ }^{\# \#} \mathrm{P}<0.01$ and ${ }^{\# \# \#} \mathrm{P}<0.001$, vs. ultrasound group. HUVECs, human umbilical vein endothelial cells; HMECs, human microvascular endothelial cells; Bcl-2, B-cell lymphoma-2; Bax, Bcl-2-associated X protein; Ctrl, control.

LIPUS-treated and control cells, the ratio of Bax to Bcl-2 was significantly increased following LIPUS treatment, which indicated high susceptibility of these cells to apoptosis. These results suggested that the mitochondrial intrinsic pathway served an important role in mediating LIPUS-induced apoptosis in HUVECs.

ERK, JNK and p38 are members of the MAPK family of signaling proteins that are involved in the initiation of apoptosis. These proteins are activated by extracellular stimuli, including mitogens, ultraviolet irradiation, heat shock, osmotic stress and cytokines, and exert various cellular effects, such as differentiation, proliferation, autophagy and apoptosis (24,33-35). The p38 pathway is known to regulate tumor cells in multiple ways; however, despite the tumor-suppressive and anti-proliferative properties of p38 in certain tissues, the p38 pathway also exerts oncogenic effects by influencing cancer metabolism and chemoresistance in cancer tissues (36). Several chemotherapeutic agents require 

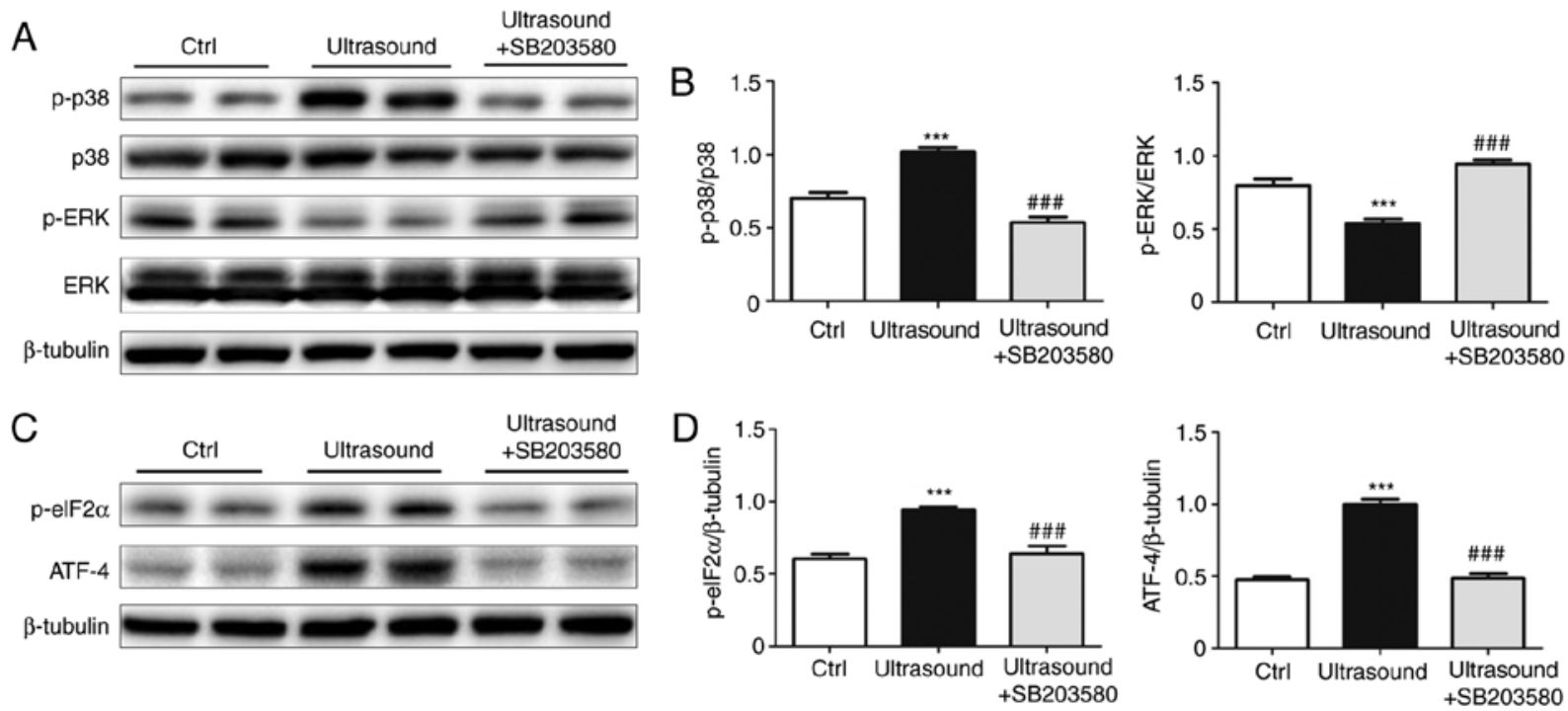

Figure 5. Low-intensity pulsed ultrasound-induced apoptosis was dependent on p38 phosphorylation. (A) Western blots and (B) quantified protein levels of p-p38 and p-ERK in HUVECs treated with S203580, normalized to the corresponding total protein levels. (C) Western blots and (D) quantified protein levels of p-eIF2 $\alpha$ and ATF-4 in HUVECs treated with S203580, normalized to $\beta$-tubulin. All values are expressed as the mean \pm standard error of three independent experiments. ${ }^{* * *} \mathrm{P}<0.001$ vs. Ctrl group; ${ }^{\# \# \#} \mathrm{P}<0.001$ vs. ultrasound group. HUVECs, human umbilical vein endothelial cells; ERK, extracellular signal-regulated kinase; ATF-4, activating transcription factor-4; eIF2 $\alpha$, eukaryotic initiation factor $2 \alpha$; p-, phosphorylated; Ctrl, control.

p38 activity for the induction of apoptosis (37). For instance, cyclophosphamide, a commonly used chemotherapeutic drug for breast cancer, induces apoptosis via activation of the $\mathrm{p} 38$ MAPK pathway (38). The present study demonstrated that a pro-apoptotic dose of LIPUS led to the activation of p38 and the inhibition of ERK, while inhibition of p38 phosphorylation reversed the pro-apoptotic and anti-angiogenic effects of LIPUS. No significant change in the levels of JNK was observed when cells were exposed to LIPUS. Activation of JNK mainly occurs due to endogenous chemical stimuli, such as DNA damage and inflammatory cytokines (39). JNK can also be activated by mechanical stimulation $(40,41)$. However, the present study data suggested that JNK may not be involved in LIPUS-induced apoptosis in HUVECs. Our previously study reported that a lower dose of LIPUS with an ultrasonic intensity of $109.4 \mathrm{~mW} / \mathrm{cm}^{2}$ promoted apoptosis in rat preadipocytes via activation of $\mathrm{p} 38$ (42). These results suggested a universal role for $\mathrm{p} 38$ in mediating LIPUS-induced apoptosis, independent of cell type.

Previous studies have demonstrated that $\mathrm{p} 38$ signaling controls the expression of several ER stress proteins by regulating the activity of transcription factors, such as ATF-1, ATF-2, C/EBP homologous protein (CHOP) and multiple cyclic AMP response element-binding proteins $(33,43)$. CHOP is an ER-specific pro-apoptotic transcription factor that is induced by growth arrest and DNA damage, and CHOP expression is regulated by the eIF2 $\alpha /$ ATF- 4 pathway $(22,44)$. Regulation of the eIF2 $\alpha / \mathrm{ATF}-4$ pathway by $\mathrm{p} 38$ has also been reported to cause apoptosis via autophagy (43). The current study results demonstrated that LIPUS treatment resulted in increased expression of p-eIF $2 \alpha$ and ATF- 4 , and that p38 inhibition reversed the upregulation of p-eIF $2 \alpha$ and ATF- 4 levels. However, no change was observed in the expression levels of LC3B, an autophagy protein, in LIPUS-treated cells. These findings suggested that $\mathrm{p} 38$ served a key role in LIPUS-induced apoptosis via activation of the ER stress response without affecting autophagy. The detailed mechanism by which $\mathrm{p} 38$ mediates eIF2 $\alpha /$ ATF- 4 upregulation is a subject for further study.

However, there are certain limitations to the current study, which should be taken into consideration. Firstly, the experiments were performed in HUVECs and HMECs rather than primary endothelial cells isolated from tumor tissues. Since obtaining sufficient numbers of the primary tumor vascular endothelial cells is almost impossible, it was speculated that the artificially-induced endothelial cells differentiated from tumor stem cells may act as an alternative approach. Furthermore, the treatment strategy and indicated dose of ultrasound were produced under in vitro conditions. Whether the dose of ultrasound is applicable for in vivo anti-angiogenesis therapy in solid tumors requires further investigation in animal tumor models.

In conclusion, the results of the present study revealed that LIPUS promoted apoptosis in HUVECs via p38 MAPK-mediated activation of the ER stress response. The findings suggested that LIPUS is an effective and low-risk option for inhibiting endothelial cell function, and this technique can potentially be used as an anti-angiogenic therapy in tumor treatment.

\section{Acknowledgements}

Not applicable.

\section{Funding}

This study was supported by grants from the National Natural Science Foundation of China (nos. 81627802 and 81570247), and the Priority Academic Program Development of Jiangsu Higher Education Institutions (grant no. PAPD 2014-2016). 


\section{Availability of data and materials}

All data generated or analyzed during the present study are included in the published article.

\section{Authors' contributions}

ZS, TX and WS designed the study and drafted the manuscript. XG, JT and DZ made substantial contributions to the study conception and design, data analysis and interpretation, and drafting and revising of the manuscript. YS and WS assisted with the molecular biology experiments. XK assisted with LIPUS manipulation. YS, WS and XK revised the manuscript. ZS, YW and TX performed the experiments and analyzed the data. All authors read and approved the final manuscript.

\section{Ethics approval and consent to participate}

Not applicable.

\section{Patient consent for publication}

Not applicable.

\section{Competing interests}

The authors declare that they have no competing interests.

\section{References}

1. Stylianopoulos T: The solid mechanics of cancer and strategies for improved therapy. J Biomech Eng 139, 2017.

2. Fukumura D and Jain RK: Tumor microenvironment abnormalities: Causes, consequences, and strategies to normalize. J Cell Biochem 101: 937-949, 2007.

3. Goel S, Duda DG, Xu L, Munn LL, Boucher Y, Fukumura D and Jain RK: Normalization of the vasculature for treatment of cancer and other diseases. Physiol Rev 91: 1071-1121, 2011.

4. Martin JD, Fukumura D, Duda DG, Boucher Y and Jain RK: Reengineering the tumor microenvironment to alleviate hypoxia and overcome cancer heterogeneity. Cold Spring Harb Perspect Med 6, 2016.

5. Jain RK: Normalizing tumor microenvironment to treat cancer: Bench to bedside to biomarkers. J Clin Oncol 31: 2205-2218, 2013

6. Vassilakopoulou M, Psyrri A and Argiris A: Targeting angiogenesis in head and neck cancer. Oral Oncol 51: 409-415, 2015.

7. Tas SW, Maracle CX, Balogh E and Szekanecz Z: Targeting of proangiogenic signalling pathways in chronic inflammation. Nat Rev Rheumatol 12: 111-122, 2016.

8. Simon T, Gagliano T and Giamas G: Direct effects of anti-angiogenic therapies on tumor cells: VEGF signaling. Trends Mol Med 23: 282-292, 2017.

9. Ye W: The complexity of translating anti-angiogenesis therapy from basic science to the clinic. Dev Cell 37: 114-125, 2016.

10. Al-Bataineh O, Jenne J and Huber P: Clinical and future applications of high intensity focused ultrasound in cancer. Cancer Treat Rev 38: 346-353, 2012.

11. Kennedy JE: High-intensity focused ultrasound in the treatment of solid tumours. Nat Rev Cancer 5: 321-327, 2005.

12. Brown MR, Farquhar-Smith P, Williams JE, ter Haar G and deSouza NM: The use of high-intensity focused ultrasound as a novel treatment for painful conditions-a description and narrative review of the literature. Br J Anaesth 115: 520-530, 2015.

13. Pan $\mathrm{H}$, Zhou W and Wang S: Pulsed focused ultrasound stimulates the release of tumor biomarkers into the blood circulation. Radiology 285: 1058-1060, 2017.

14. Hariharan P, Myers MR and Banerjee RK: HIFU procedures at moderate intensities-effect of large blood vessels. Phys Med Biol 52: 3493-3513, 2007.
15. Qiao Y, Yin H, Li Z and Wan M: Cavitation distribution within large phantom vessel and mechanical damage formed on surrounding vessel wall. Ultrason Sonochem 20: 1376-1383, 2013.

16. Mizrahi N, Zhou EH, Lenormand G, Krishnan R, Weihs D, Butler JP, Weitz DA, Fredberg JJ and Kimmel E: Low intensity ultrasound perturbs cytoskeleton dynamics. Soft matter 8: 2438-2443, 2012.

17. Rutten S, Nolte PA,Korstjens CM and Klein-Nulend J: Low-intensity pulsed ultrasound affects RUNX2 immunopositive osteogenic cells in delayed clinical fracture healing. Bone 45: 862-869, 2009.

18. Hitchcock KE and Holland CK: Ultrasound-assisted thrombolysis for stroke therapy: Better thrombus break-up with bubbles. Stroke 41: S50-S53, 2010.

19. Zhang Z, Chen J, Chen L, Yang X, Zhong H, Qi X, Bi Y and $\mathrm{Xu}$ K: Low frequency and intensity ultrasound induces apoptosis of brain glioma in rats mediated by caspase-3, Bcl-2, and survivin. Brain Res 1473: 25-34, 2012.

20. Zhou XY, Wu SY, Zhang ZC, Wang F, Yang YL, Li M and Wei XZ: Low-intensity pulsed ultrasound promotes endothelial cell-mediated osteogenesis in a conditioned medium coculture system with osteoblasts. Medicine (Baltimore) 96: e8397, 2017.

21. Marquez RT and Xu L: Bcl-2: Beclin 1 complex: Multiple, mechanisms regulating autophagy/apoptosis toggle switch. Am J Cancer Res 2: 214-221, 2012.

22. Rovetta F, Stacchiotti A, Consiglio A, Cadei M, Grigolato PG, Lavazza A, Rezzani R and Aleo MF: ER signaling regulation drives the switch between autophagy and apoptosis in NRK-52E cells exposed to cisplatin. Exp Cell Res 318: 238-250, 2012.

23. Furusawa Y, Zhao QL, Hassan MA, Tabuchi Y, Takasaki I, Wada S and Kondo T: Ultrasound-induced apoptosis in the presence of Sonazoid and associated alterations in gene expression levels: A possible therapeutic application. Cancer Lett 288: 107-115, 2010.

24. Gao Q, Walmsley AD, Cooper PR and Scheven BA: Ultrasound stimulation of different dental stem cell populations: Role of mitogen-activated protein kinase signaling. J Endod 42: 425-431, 2016.

25. Lee GS, Park JH, Shin US and Kim HW: Direct deposited porous scaffolds of calcium phosphate cement with alginate for drug delivery and bone tissue engineering. Acta Biomater 7: 3178-3186, 2011.

26. Mason TJ: Therapeutic ultrasound an overview. Ultrason Sonochem 18: 847-852, 2011.

27. Hou R, Xu Y, Lu Q, Zhang Y and Hu B: Effect of low-frequency low-intensity ultrasound with microbubbles on prostate cancer hypoxia. Tumour Biol 39: 1010428317719275, 2017.

28. Chen H, Brayman AA, Bailey MR and Matula TJ: Blood vessel rupture by cavitation. Urol Res 38: 321-326, 2010.

29. Elmore S: Apoptosis: A review of programmed cell death. Toxicol Pathol 35: 495-516, 2007.

30. Chen Y and Brandizzi F: IRE1: ER stress sensor and cell fate executor. Trends Cell Biol 23: 547-555, 2013.

31. Bhutia SK, Dash R, Das SK, Azab B, Su ZZ, Lee SG, Grant S, Yacoub A, Dent P, Curiel DT, et al: Mechanism of autophagy to apoptosis switch triggered in prostate cancer cells by antitumor cytokine melanoma differentiation-associated gene 7/interleukin-24. Cancer Res 70: 3667-3676, 2010.

32. Wu H, Che X, Zheng Q, Wu A, Pan K, Shao A, Wu Q, Zhang J and Hong Y: Caspases: A molecular switch node in the crosstalk between autophagy and apoptosis. Int J Biol Sci 10: 1072-1083, 2014.

33. Obata T, Brown GE and Yaffe MB: MAP kinase pathways activated by stress: The p38 MAPK pathway. Crit Care Med 28: N67-N77, 2000.

34. Whitmarsh AJ: A central role for p38 MAPK in the early transcriptional response to stress. BMC Biol 8: 47, 2010.

35. Trempolec N, Dave-Coll N and Nebreda AR: SnapShot: p38 MAPK substrates. Cell 152: 924-924.e1, 2013.

36. Grossi V, Peserico A, Tezil T and Simone C: p38alpha MAPK pathway: A key factor in colorectal cancer therapy and chemoresistance. World J Gastroenterol 20: 9744-9758, 2014.

37. Olson JM and Hallahan AR: p38 MAP kinase: A convergence point in cancer therapy. Trends Mol Med 10: 125-129, 2004.

38. Pang H, Cai L, Yang Y, Chen X, Sui G and Zhao C: Knockdown of osteopontin chemosensitizes MDA-MB-231 cells to cyclophosphamide by enhancing apoptosis through activating p38 MAPK pathway. Cancer Biother Radiopharm 26: 165-173, 2011.

39. Sui X, Kong N, Ye L, Han W, Zhou J, Zhang Q, He C and Pan H: p38 and JNK MAPK pathways control the balance of apoptosis and autophagy in response to chemotherapeutic agents. Cancer Lett 344: 174-179, 2014 
40. Matsui H, Fukuno N, Kanda Y, Kantoh Y, Chida T, Nagaura Y, Suzuki O, Nishitoh $\mathrm{H}$, Takeda K, Ichijo $\mathrm{H}$, et al: The expression of Fn14 via mechanical stress-activated JNK contributes to apoptosis induction in osteoblasts. J Biol Chem 289: 6438-6450, 2014.

41. Mu C,Lv T, Wang Z, Ma S, Ma J, Liu J, Yu J and Mu J: Mechanical stress stimulates the osteo/odontoblastic differentiation of human stem cells from apical papilla via erk $1 / 2$ and JNK MAPK pathways. Biomed Res Int 2014: 494378, 2014.

42. Xu T, Gu J, Li C, Guo X, Tu J, Zhang D, Sun W and Kong X: Low-intensity pulsed ultrasound suppresses proliferation and promotes apoptosis via $\mathrm{p} 38$ MAPK signaling in rat visceral preadipocytes. Am J Transl Res 10: 948-956, 2018.
43. Jiang Q, Li F, Shi K, Wu P, An J, Yang Y and Xu C: Involvement of p38 in signal switching from autophagy to apoptosis via the PERK/eIF2 $\alpha / \mathrm{ATF}-4$ axis in selenite-treated NB4 cells. Cell Death Dis 5: e1270, 2014

44. Rozpedek W, Pytel D, Mucha B, Leszczynska H, Diehl JA and Majsterek I: The role of the PERK/eIF2 $\alpha / \mathrm{ATF}-4 / \mathrm{CHOP}$ signaling pathway in tumor progression during endoplasmic reticulum stress. Curr Mol Med 16: 533-544, 2016.

(i) (3) This work is licensed under a Creative Commons Attribution-NonCommercial-NoDerivatives 4.0 International (CC BY-NC-ND 4.0) License. 